Terr. Atmos. Ocean. Sci., Vol. 18, No. 5, 951-973, December 2007

\title{
Relationships Among Magnitudes and Seismic Moment of Earthquakes in the Taiwan Region
}

\author{
Kou-Cheng Chen ${ }^{1,}{ }^{*}$, Win-Gee Huang ${ }^{1}$, and Jeen-Hwa Wang ${ }^{1}$ \\ (Manuscript received 6 March 2007, in final form 21 June 2007)
}

\begin{abstract}
The seismic moments $\left(M_{0}\right)$, body-wave magnitudes $\left(m_{b}\right)$, and surfacewave magnitudes $\left(M_{s}\right)$ of 201 Taiwan earthquakes with $4.8 \leq m_{b} \leq 6.6$ published in the Global CMT catalog from 1976 to 2006 are used to study the correlations among the three source parameters. The resultant relationships are: $\log \left(M_{0}\right)=(1.07 \pm 0.04) M_{s}+(18.72 \pm 0.20) ; \log \left(M_{0}\right)=(1.73 \pm$ $0.09) m_{b}+(15.09 \pm 0.52)$; and $M_{s}=(1.46 \pm 0.08) m_{b}-(2.52 \pm 0.43)$. The three relationships have high agreement with those of earthquakes in the circum-Pacific seismic belt. This might imply that the tectonic conditions and source properties of the Taiwan region behave like the average ones of the circum-Pacific seismic belt. The relationships between the three source parameters and local magnitude are: $\log \left(M_{0}\right)=(1.27 \pm 0.06) M_{L}+(17.23 \pm$ $0.35) ; m_{b}=(0.66 \pm 0.03) M_{L}+(1.69 \pm 0.17) ;$ and $M_{s}=(1.03 \pm 0.06) M_{L}-$ $(0.53 \pm 0.36)$.
\end{abstract}

(Key words: Seismic moment, Magnitude scales, Relationship)

\section{INTRODUCTION}

Magnitude is regarded as the most directly measurable and simple parameter to specify quantitatively the size of an earthquake. Since Richter (1935) first defined the local magnitude, $\mathrm{M}_{\mathrm{L}}$, several magnitude scales have been proposed. The surface-wave magnitude $\left(\mathrm{M}_{\mathrm{s}}\right)$ and bodywave magnitude $\left(\mathrm{m}_{\mathrm{b}}\right)$ are two commonly-used scales. From the study of source mechanism by an elastic dislocation theory, Aki $(1966,1967)$ stated that the amplitude of a very long period wave is proportional to the seismic moment, $M_{0}$, of an earthquake. Aki (1966) first measured the value of $\mathrm{M}_{\mathrm{o}}$ of the 1964 Niigata, Japan, earthquake. Ben-Menahem et al. (1969) also suggested

\footnotetext{
${ }^{1}$ Institute of Earth Sciences, Academia Sinica, Taipei, Taiwan, ROC

* Corresponding author address: Dr. Kou-Cheng Chen, Institute of Earth Sciences, Academia Sinica, Taipei, Taiwan, ROC; E-mail: chenkc@earth.sinica.edu.tw doi: 10.3319/TAO.2007.18.5.951(T)
} 
that the far-field static-strain field is proportional to $\mathrm{M}_{\mathrm{o}}$. From then on, seismic moment was considered a new parameter to specify the size of an earthquake. Based on $\mathrm{M}_{\mathrm{o}}$, moment magnitude has been defined by Hanks and Kanamori (1979) since 1979.

$\mathrm{M}_{\mathrm{L}}, \mathrm{M}_{\mathrm{s}}, \mathrm{m}_{\mathrm{b}}$, and $\mathrm{M}_{\mathrm{o}}$ are estimated from seismic waves of different types and periods (cf. Miyamura 1978): $\mathrm{M}_{\mathrm{L}}$ from the maximum trace amplitude in millimeters recorded on a WoodAnderson standard torsion seismograph, $\mathrm{M}_{\mathrm{s}}$ from teleseismic surface waves in the period range of $17-23 \mathrm{sec}, \mathrm{m}_{\mathrm{b}}$ from teleseismic $\mathrm{P}$-waves in the period range of $0.1-3 \mathrm{sec}$., and $\mathrm{M}_{\mathrm{o}}$ from mantle waves, a very long period range. Various magnitude scales will sample different information of the radiated spectrum generated from the fracture process of an earthquake. Miyamura (1982) stated that combined use of different magnitude scales will give better description of the nature of an earthquake and seismicity. Nortman and Duda (1983) argued that the large variety of magnitude scales indicates that the strength of an earthquake needs to be determined from various parts of the radiated spectrum.

There are some relationships among source parameters (Chung and Bernreuter 1981; Wang 1992). This concept can be illustrated by the scaling law proposed by Aki (1967). Aki (1967) first studied the correlation between $\mathrm{M}_{\mathrm{s}}$ and $\mathrm{m}_{\mathrm{b}}$, which is the original body-wave magnitude defined by Gutenberg (1945), constructed by Gutenberg and Richter (1956). His results showed that the $\omega^{-2}$ model proposed by Aki (1967) is better than the $\omega^{-3}$ model suggested by Haskell (1964). The two source spectral models are briefly described below: at low frequencies, the spectral amplitude is almost proportional to the seismic moment; while at high frequencies, especially for higher than the corner frequency, the spectral amplitude is proportional to $\omega^{-2}$ for the former and $\omega^{-3}$ for the latter. Aki (1972) constructed the theoretical correlation for $\log \left(M_{0}\right)$ versus $\mathrm{M}_{\mathrm{s}}$. His results show that $\log \left(\mathrm{M}_{\mathrm{o}}\right)$ exponentially increase with $\mathrm{M}_{\mathrm{s}}$. Of course, his correlation can be simplified to be: $\log \left(\mathrm{M}_{\mathrm{o}}\right) \sim 3 \mathrm{M}_{\mathrm{s}}$ as $\mathrm{M}_{\mathrm{s}}>7$ and $\log \left(\mathrm{M}_{\mathrm{o}}\right) \sim \mathrm{M}_{\mathrm{s}}$ as $\mathrm{M}_{\mathrm{s}}<7$. Kanamori and Anderson (1975) studied the correlation between these based on the $\omega^{-2}$ model, with a more reliable relation between fault length and duration time than that suggested by Aki (1972). Their results show $\log \left(\mathrm{M}_{\mathrm{o}}\right) \sim 1.5 \mathrm{M}_{\mathrm{s}}$ for common earthquakes and $\log \left(\mathrm{M}_{\mathrm{o}}\right) \sim 3 \mathrm{M}_{\mathrm{s}}$ for some events with long duration times. Geller (1976) studied the correlations of $M_{o}$ versus $M_{s}$ and $M_{s}$ versus $\mathrm{m}_{\mathrm{b}}$ based on the $\omega^{-3}$ model. His results show the dependence of the correlations upon magnitude range. Ekstrom and Dziewonski (1988) obtained the empirical relationship of $\log \left(\mathrm{M}_{\mathrm{o}}\right)$ versus global earthquakes: (1) $\log \left(\mathrm{M}_{\mathrm{o}}\right)=19.24+\mathrm{M}_{\mathrm{s}}$ for $\mathrm{M}_{\mathrm{s}} \leq 5.3$; (2) $\log \left(\mathrm{M}_{\mathrm{o}}\right)=30.20-(92.45-$ $\left.11.40 \mathrm{M}_{\mathrm{s}}\right)^{1 / 2}$ for $5.3 \leq \mathrm{M}_{\mathrm{s}} \leq 6.8$; and (3) $\log \left(\mathrm{M}_{\mathrm{o}}\right)=16.14+1.5 \mathrm{M}_{\mathrm{s}}$ for $\mathrm{M}_{\mathrm{s}} \geq 6.8$. Koyama (1994) obtained theoretical correlations among the three parameters: (1) $\log \left(\mathrm{M}_{\mathrm{o}}\right) \sim \mathrm{M}_{\mathrm{s}}$ for $\mathrm{M}_{\mathrm{s}}<7.5$ and $\log \left(\mathrm{M}_{\mathrm{o}}\right) \sim 2 \mathrm{M}_{\mathrm{s}}$ for $\mathrm{M}_{\mathrm{s}}>7.5$; (2) $\log \left(\mathrm{M}_{\mathrm{o}}\right) \sim 2.5 \mathrm{~m}_{\mathrm{b}}$.

However, the relationships should be of regional dependence (cf. Chung and Bernreuter 1981). The interrelations among various source parameters may display regional characteristics. A particularly significant problem is to discriminate different tectonic provinces by the use of the above-mentioned concept. In a series of papers, Nuttli (1983a, b, c) found distinction between the average source parameter relations for mid-plate earthquakes and those for platemargin earthquakes.

Because Taiwan is situated at the collision boundary between the Eurasian plate and the Philippine Sea plate (Tsai et al. 1977; Wu 1978; Lin 2002), the earthquakes occurring in this region should have plate-margin characteristics. Wang (1985) estimated the relationships of 
$M_{o}$ versus $M_{s}, M_{o}$ versus $m_{b}$, and $M_{s}$ versus $m_{b}$ for 16 earthquakes in the Taiwan region, and stated that the relationships for regional events are similar to those for global earthquakes inferred by Nuttli (1985). Yeh et al. (1982) determined the local magnitude from the maximum amplitude of the Wood-Anderson-type seismograms simulated from accelerograms. Yeh and Hsu (1985) determined the local magnitude from the maximum amplitude of the seismogram recorded by a simulated Wood-Anderson seismograph operated by the Institute of Earth Sciences (IES), Academia Sinica (Liu 1981). They related this local magnitude to duration magnitude. Cheng and Yeh (1989) related this local magnitude to $\mathrm{m}_{\mathrm{b}}$ for 143 events that occurred from 1873 to 1985 . Wang et al. (1989) determined the local magnitude from the maximum amplitude of the seismogram recorded by the same simulated Wood-Anderson seismograph and also related the local magnitude to $\mathrm{M}_{\mathrm{o}}$ and $\mathrm{m}_{\mathrm{b}}$ determined from teleseismic data. Li and Chiu (1989) correlated seismic moment to local magnitude for earthquakes in Taiwan. Wang (1998) reviewed numerous relationships among seismic moment and earthquake magnitudes. Shin (1993) determined the local magnitude from the maximum amplitudes of the Wood- Anderson-type seismograms simulated from short-period seismograms of the Central Weather Bureau Seismic Network (CWBSN). Since 1991, this local magnitude has been used by CWBSN to quantify earthquakes in Taiwan. Although this local magnitude is not defined based on a standard Wood-Anderson seismograph, it is still denoted by $\mathrm{M}_{\mathrm{L}}$ below.

However, the relationships inferred by Wang (1985) were just based on a small data set. Since 1985 more earthquakes have occurred in the Taiwan region. To understand the general characteristics of the source spectra of earthquakes, it is necessary to investigate the relationships again. Therefore, in this study these relationships will be inferred by using more data published in the Earthquake Data Report (EDR) of the United States Geological Survey (USGS). Since September 1991 the local magnitude of earthquakes has been routinely determined by the CWBSN (Shin 1993). Hence, it is significant to investigate the relationships of $\mathbf{M}_{\mathrm{o}}$ versus $M_{L}, m_{b}$ versus $M_{L}$, and $M_{s}$ versus $M_{L}$.

\section{DATA}

Since 1968, the body-wave magnitudes and surface-wave magnitudes have been routinely determined by the USGS for large earthquakes around the world. Since October 1982 the seismic moment has been also provided in the EDR of the USGS for larger-sized earthquakes. Since the summer of 2006, the main activities of the Harvard CMT Project have been under "The Global CMT Project." The CMT solutions and the best double couples are published at the web site http://www.globalcmt.org/. Since September 1991, the CWBSN has routinely estimated the local magnitude of earthquakes from simulated Wood-Anderson seismograms, which are produced from digital three-component short-period seismograms (Shin 1993). A total of 209 earthquakes in the Taiwan region, ranging from 119 to $123.5^{\circ} \mathrm{E}$ and 21 to $26^{\circ} \mathrm{N}$, during the period of January 1977 to October 2006 are selected from the Global CMT catalog. The selected data are compared with those events located by the CWBSN. Eight distant events are excluded because they are not located by the CWBSN. The related source parameters of 201 earthquakes are $4.2 \leq \mathrm{M}_{\mathrm{s}} \leq 7.8 ; 4.8 \leq \mathrm{m}_{\mathrm{b}} \leq 6.6 ; 4.6 \leq \mathrm{M}_{\mathrm{L}} \leq 7.3$; and $1.4 \times 10^{23}$ dyne $-\mathrm{cm} \leq$ $\mathrm{M}_{\mathrm{o}} \leq 3.38 \times 10^{27}$ dyne $-\mathrm{cm}$. The focal depths range from 0.5 to $61 \mathrm{~km}$. Earthquake locations as 
well as focal depths taken from the CWBSN catalog and source parameters are listed in Table 1. The columns denoted by ' $\mathrm{M}_{\mathrm{o}}$ ', ' $\mathrm{m}_{\mathrm{b}}$ ', ' $\mathrm{M}_{\mathrm{s}}$ ', and ' $\mathrm{M}_{\mathrm{L}}$ ' present the seismic moment, body-wave magnitude, surface-wave magnitude, and local magnitude, respectively. Figure 1 shows their epicenters. Most events are located in eastern and offshore Taiwan.

Table 1. Seismic source parameters of 201 moderate Taiwan earthquakes during January 1977 to October 2006. The data are selected from the Global CMT catalog of the United States Geological Survey. The unit of $\mathrm{M}_{\mathrm{o}}$ is $10^{25}$ dyne-cm.

\begin{tabular}{ccccccccc}
\hline No. & Time & $\begin{array}{c}\text { Latitude } \\
\left({ }^{\mathbf{0}}\right)\end{array}$ & $\begin{array}{c}\text { Longitude } \\
\left({ }^{\mathbf{0}} \mathbf{E}\right)\end{array}$ & $\begin{array}{c}\text { Depth } \\
(\mathbf{k m})\end{array}$ & $\mathbf{m}_{\mathbf{b}}$ & $\mathbf{M}_{\mathbf{s}}$ & $\mathbf{M}_{\mathbf{L}}$ & $\mathbf{M}_{\mathbf{0}}$ \\
\hline 1 & $1977 / 01 / 07 / 19: 36$ & 21.81 & 120.25 & 5.00 & 5.7 & 5.1 & - & 0.388 \\
2 & $1977 / 07 / 15 / 02: 12$ & 23.96 & 122.38 & 0.60 & 5.5 & 5.7 & - & 0.681 \\
3 & $1978 / 02 / 08 / 00: 15$ & 23.92 & 122.70 & 5.00 & 5.5 & 5.7 & - & 1.4 \\
4 & $1978 / 03 / 14 / 20: 32$ & 23.90 & 122.78 & 6.62 & 5.5 & 5.4 & - & 0.671 \\
5 & $1978 / 04 / 29 / 19: 25$ & 24.65 & 122.77 & 14.00 & 5.4 & 5.8 & - & 0.845 \\
6 & $1978 / 07 / 23 / 14: 42$ & 22.35 & 121.33 & 6.10 & 6.5 & 7.4 & - & 86.4 \\
7 & $1978 / 08 / 09 / 18: 35$ & 23.50 & 121.50 & 13.04 & 5.2 & 5.5 & - & 0.171 \\
8 & $1978 / 09 / 10 / 16: 34$ & 24.04 & 121.76 & 3.06 & 5.4 & 5.1 & - & 0.248 \\
9 & $1978 / 12 / 23 / 11: 23$ & 23.30 & 122.00 & 4.12 & 6.6 & 7.0 & - & 35.6 \\
10 & $1979 / 12 / 02 / 05: 25$ & 22.96 & 121.41 & 19.05 & 5.5 & 5.7 & - & 0.804 \\
11 & $1980 / 10 / 18 / 00: 08$ & 24.26 & 121.89 & 10.00 & 5.0 & 5.5 & - & 0.225 \\
12 & $1980 / 11 / 07 / 12: 36$ & 23.96 & 122.30 & 24.28 & 5.4 & 5.4 & - & 0.581 \\
13 & $1981 / 01 / 29 / 04: 51$ & 24.42 & 121.91 & 12.81 & 5.6 & 5.5 & - & 0.968 \\
14 & $1981 / 02 / 20 / 20: 09$ & 22.91 & 121.43 & 21.26 & 5.4 & 5.7 & - & 0.071 \\
15 & $1981 / 03 / 02 / 12: 13$ & 22.95 & 121.43 & 14.30 & 5.5 & 5.9 & - & 1.48 \\
16 & $1982 / 01 / 23 / 14: 10$ & 23.91 & 121.63 & 3.25 & 5.6 & 6.0 & - & 1.307 \\
17 & $1982 / 10 / 20 / 20: 01$ & 23.77 & 121.96 & 3.13 & 5.4 & 5.9 & - & 0.837 \\
18 & $1983 / 05 / 10 / 00: 15$ & 24.46 & 121.51 & 1.23 & 5.7 & 5.4 & - & 0.427 \\
19 & $1983 / 06 / 21 / 14: 48$ & 23.90 & 122.57 & 14.77 & 5.8 & 6.3 & - & 2.58 \\
20 & $1983 / 06 / 25 / 19: 40$ & 23.82 & 122.64 & 16.28 & 5.5 & 5.0 & - & 0.174 \\
21 & $1983 / 09 / 07 / 23: 11$ & 23.90 & 122.49 & 21.68 & 5.5 & 5.7 & - & 0.265 \\
\hline
\end{tabular}


Table 1. (Continued)

\begin{tabular}{|c|c|c|c|c|c|c|c|c|}
\hline No. & Time & $\begin{array}{c}\text { Latitude } \\
\left({ }^{0} \mathbf{N}\right)\end{array}$ & $\begin{array}{c}\text { Longitude } \\
\left({ }^{0} \mathrm{E}\right)\end{array}$ & $\begin{array}{c}\text { Depth } \\
(\mathbf{k m})\end{array}$ & $\mathbf{m}_{\mathbf{b}}$ & $\mathbf{M}_{\mathrm{s}}$ & $\mathbf{M}_{\mathrm{L}}$ & $\mathbf{M}_{\mathbf{o}}$ \\
\hline 22 & 1983/09/09/17:01 & 23.92 & 122.60 & 15.88 & 5.3 & 5.1 & - & 0.3 \\
\hline 23 & $1983 / 09 / 21 / 19: 20$ & 23.94 & 122.32 & 18.00 & 6.0 & 6.4 & - & 5.06 \\
\hline 24 & $1983 / 09 / 23 / 12: 29$ & 23.89 & 122.35 & 12.32 & 5.7 & 5.9 & - & 1.102 \\
\hline 25 & 1983/10/07/20:05 & 23.84 & 122.66 & 17.69 & 5.1 & 4.7 & - & 0.048 \\
\hline 26 & $1984 / 01 / 19 / 11: 12$ & 24.04 & 122.49 & 21.62 & 5.1 & 5.2 & - & 0.065 \\
\hline 27 & 1984/03/28/09:11 & 23.88 & 122.78 & 10.30 & 5.5 & 5.9 & - & 0.88 \\
\hline 28 & $1984 / 04 / 19 / 17: 29$ & 24.82 & 122.24 & 6.10 & 5.2 & 5.0 & - & 0.081 \\
\hline 29 & 1984/12/29/01:06 & 24.80 & 122.02 & 60.74 & 5.4 & 5.0 & - & 0.284 \\
\hline 30 & $1985 / 01 / 13 / 21: 51$ & 24.01 & 122.22 & 44.15 & 5.8 & 5.3 & - & 0.173 \\
\hline 31 & $1985 / 02 / 18 / 19: 41$ & 23.44 & 123.00 & 10.33 & 5.7 & 5.6 & - & 0.315 \\
\hline 32 & $1985 / 06 / 12 / 17: 22$ & 24.57 & 122.19 & 3.31 & 5.2 & 5.8 & - & 0.772 \\
\hline 33 & 1985/09/20/15:01 & 24.53 & 122.20 & 6.11 & 5.3 & 5.0 & - & 0.18 \\
\hline 34 & 1986/01/16/13:04 & 24.76 & 121.96 & 10.22 & 5.5 & 5.9 & - & 1.071 \\
\hline 35 & $1986 / 02 / 27 / 06: 23$ & 23.91 & 122.47 & 7.20 & 5.8 & 5.4 & - & 0.568 \\
\hline 36 & $1986 / 03 / 22 / 04: 45$ & 23.36 & 121.74 & 28.45 & 5.6 & 5.1 & - & 0.847 \\
\hline 37 & $1986 / 03 / 22 / 10: 31$ & 24.75 & 122.92 & 33.00 & 5.2 & 5.7 & - & 0.285 \\
\hline 38 & $1986 / 03 / 22 / 12: 06$ & 24.76 & 122.20 & 7.08 & 5.3 & 5.7 & - & 0.535 \\
\hline 39 & $1986 / 03 / 22 / 14: 27$ & 24.56 & 122.61 & 1.25 & 4.9 & 5.5 & - & 0.126 \\
\hline 40 & $1986 / 03 / 25 / 12: 13$ & 24.76 & 122.66 & 5.90 & 5.1 & 5.0 & - & 0.063 \\
\hline 41 & $1986 / 05 / 20 / 05: 25$ & 24.08 & 121.59 & 15.82 & 6.2 & 6.3 & - & 2.37 \\
\hline 42 & $1986 / 06 / 04 / 16: 21$ & 23.94 & 121.68 & 1.41 & 5.2 & 5.3 & - & 0.097 \\
\hline 43 & 1986/07/30/11:31 & 24.63 & 121.79 & 1.55 & 5.6 & 5.6 & - & 0.22 \\
\hline 44 & $1986 / 07 / 31 / 11: 36$ & 24.83 & 122.66 & 6.50 & 5.1 & 5.2 & - & 0.093 \\
\hline 45 & 1986/11/14/21:20 & 23.99 & 121.83 & 15.00 & 6.2 & 7.8 & - & 130 \\
\hline 46 & $1986 / 11 / 15 / 07: 24$ & 23.90 & 121.69 & 14.20 & 5.5 & 5.8 & - & 0.529 \\
\hline 47 & 1987/01/06/05:07 & 23.91 & 121.84 & 28.61 & 5.8 & 5.6 & - & 0.399 \\
\hline 48 & 1987/04/11/18:13 & 23.86 & 122.21 & 7.54 & 5.6 & 4.7 & - & 0.091 \\
\hline 49 & $1987 / 06 / 27 / 07: 38$ & 24.32 & 121.63 & 0.48 & 5.2 & 4.9 & - & 0.063 \\
\hline
\end{tabular}


Table 1. (Continued)

\begin{tabular}{|c|c|c|c|c|c|c|c|c|}
\hline No. & Time & $\begin{array}{c}\text { Latitude } \\
\left({ }^{0} \mathrm{~N}\right)\end{array}$ & $\begin{array}{c}\text { Longitude } \\
\left({ }^{\circ} \mathrm{E}\right)\end{array}$ & $\begin{array}{c}\text { Depth } \\
(\mathbf{k m})\end{array}$ & $\mathbf{m}_{\mathbf{b}}$ & $\mathbf{M}_{\mathbf{s}}$ & $\mathbf{M}_{\mathbf{L}}$ & $\mathbf{M}_{\mathbf{0}}$ \\
\hline 50 & $1987 / 12 / 18 / 05: 53$ & 23.38 & 120.78 & 1.92 & 5.0 & 5.3 & - & 0.083 \\
\hline 51 & 1988/02/12/19:15 & 23.81 & 122.40 & 7.13 & 5.6 & 5.3 & - & 0.342 \\
\hline 52 & 1988/04/07/03:05 & 23.98 & 121.63 & 1.35 & 5.2 & 4.5 & - & 0.332 \\
\hline 53 & 1988/04/24/20:03 & 23.45 & 122.01 & 18.40 & 5.6 & 5.5 & - & 0.166 \\
\hline 54 & $1988 / 07 / 20 / 23: 15$ & 23.91 & 121.73 & 32.29 & 5.8 & 5.6 & - & 0.814 \\
\hline 55 & $1988 / 08 / 09 / 16: 51$ & 24.12 & 122.49 & 29.89 & 5.3 & 5.0 & - & 0.061 \\
\hline 56 & $1988 / 08 / 11 / 03: 40$ & 22.15 & 121.27 & 19.60 & 5.4 & 4.8 & - & 0.15 \\
\hline 57 & 1988/10/16/12:09 & 22.02 & 121.83 & 0.75 & 5.3 & 5.0 & - & 1.332 \\
\hline 58 & 1989/08/03/11:31 & 23.08 & 122.01 & 5.61 & 5.9 & 6.3 & - & 4.83 \\
\hline 59 & $1989 / 08 / 21 / 23: 12$ & 23.96 & 122.44 & 8.12 & 5.6 & 6.2 & - & 2.82 \\
\hline 60 & 1989/09/23/17:51 & 22.54 & 122.00 & 13.68 & 5.5 & 5.0 & - & 0.196 \\
\hline 61 & 1990/07/16/19:14 & 24.18 & 121.80 & 1.39 & 5.6 & 5.8 & - & 0.417 \\
\hline 62 & 1990/08/21/16:01 & 22.60 & 121.97 & 20.70 & 5.3 & 5.3 & - & 0.162 \\
\hline 63 & 1990/09/11/14:09 & 22.81 & 120.80 & 24.16 & 5.8 & 5.6 & - & 0.542 \\
\hline 64 & 1990/11/11/23:59 & 24.10 & 121.74 & 34.33 & 5.4 & 5.3 & - & 0.204 \\
\hline 65 & 1990/12/13/03:01 & 23.88 & 121.55 & 2.82 & 5.9 & 6.2 & - & 3.18 \\
\hline 66 & 1990/12/13/19:50 & 23.77 & 121.63 & 1.26 & 5.9 & 6.3 & - & 3.63 \\
\hline 67 & $1990 / 12 / 14 / 02: 37$ & 23.60 & 121.68 & 11.07 & 5.2 & 4.6 & - & 0.104 \\
\hline 68 & 1990/12/18/04:39 & 23.84 & 121.60 & 1.84 & 4.9 & 4.8 & - & 0.121 \\
\hline 69 & 1990/12/19/00:20 & 23.67 & 121.59 & 5.20 & 5.3 & 5.3 & - & 0.276 \\
\hline 70 & 1990/12/19/23:38 & 23.73 & 121.55 & 7.72 & 5.4 & 5.2 & - & 0.183 \\
\hline 71 & $1990 / 12 / 25 / 14: 21$ & 23.77 & 121.57 & 2.04 & 5.6 & 5.7 & - & 0.76 \\
\hline 72 & 1991/01/18/01:36 & 23.68 & 121.27 & 0.79 & 5.9 & 5.5 & - & 0.364 \\
\hline 73 & 1991/03/12/06:04 & 23.25 & 120.07 & 12.26 & 5.6 & 5.3 & - & 0.155 \\
\hline 74 & 1991/03/26/03:58 & 21.67 & 121.66 & 12.14 & 5.8 & 6.3 & - & 3.03 \\
\hline 75 & 1991/03/26/06:19 & 21.70 & 121.67 & 16.60 & 5.2 & 5.2 & - & 0.201 \\
\hline 76 & $1991 / 03 / 26 / 10: 24$ & 21.42 & 121.91 & 39.60 & 5.3 & 5.4 & - & 0.145 \\
\hline 77 & 1991/09/30/09:44 & 22.60 & 121.43 & 18.81 & 5.6 & 5.2 & 5.89 & 0.139 \\
\hline
\end{tabular}


Table 1. (Continued)

\begin{tabular}{|c|c|c|c|c|c|c|c|c|}
\hline No. & Time & $\begin{array}{c}\text { Latitude } \\
\qquad\left({ }^{0} \mathbf{N}\right)\end{array}$ & $\begin{array}{c}\text { Longitude } \\
\left({ }^{0} \mathrm{E}\right)\end{array}$ & $\begin{array}{c}\text { Depth } \\
(\mathbf{k m})\end{array}$ & $\mathbf{m}_{\mathbf{b}}$ & $\mathbf{M}_{\mathrm{s}}$ & $\mathbf{M}_{\mathbf{L}}$ & $\mathbf{M}_{\mathbf{0}}$ \\
\hline 78 & $1992 / 02 / 10 / 12: 38$ & 21.57 & 121.78 & 32.64 & 5.0 & 5.2 & 4.87 & 0.103 \\
\hline 79 & 1992/04/19/18:32 & 23.84 & 121.57 & 8.07 & 5.9 & 6.1 & 5.55 & 1.56 \\
\hline 80 & $1992 / 05 / 28 / 23: 19$ & 23.13 & 121.35 & 13.68 & 5.4 & 5.1 & 5.42 & 0.238 \\
\hline 81 & $1992 / 08 / 06 / 21: 29$ & 24.66 & 122.46 & 0.88 & 5.3 & 5.7 & 5.32 & 0.25 \\
\hline 82 & $1992 / 08 / 14 / 17: 26$ & 24.03 & 121.63 & 26.07 & 5.0 & 4.9 & 5.18 & 0.061 \\
\hline 83 & 1992/09/28/14:06 & 23.88 & 122.67 & 17.58 & 5.9 & 6.1 & 5.72 & 1.67 \\
\hline 84 & $1993 / 01 / 23 / 08: 59$ & 24.08 & 121.74 & 28.50 & 5.4 & 5.3 & 5.40 & 0.424 \\
\hline 85 & 1993/12/06/23:00 & 21.84 & 120.99 & 20.79 & 5.2 & 4.8 & 5.29 & 0.132 \\
\hline 86 & 1993/12/15/21:49 & 23.21 & 120.52 & 12.50 & 5.6 & 5.2 & 5.70 & 0.153 \\
\hline 87 & 1994/03/17/11:28 & 23.96 & 122.42 & 0.59 & 5.3 & 5.4 & 5.65 & 0.244 \\
\hline 88 & $1994 / 05 / 23 / 05: 36$ & 23.92 & 122.69 & 7.21 & 5.7 & 6.0 & 5.77 & 1.89 \\
\hline 89 & $1994 / 05 / 23 / 15: 16$ & 23.86 & 122.64 & 5.54 & 5.9 & 5.7 & 6.00 & 0.813 \\
\hline 90 & 1994/05/24/04:00 & 23.83 & 122.60 & 4.45 & 6.0 & 6.6 & 6.60 & 6.6 \\
\hline 91 & 1994/06/05/01:09 & 24.46 & 121.84 & 5.30 & 6.0 & 6.5 & 6.50 & 3.8 \\
\hline 92 & $1994 / 10 / 28 / 23: 51$ & 24.64 & 122.27 & 2.00 & 5.4 & 5.4 & 5.66 & 0.328 \\
\hline 93 & 1994/12/13/00:50 & 23.93 & 122.65 & 15.85 & 5.0 & 4.6 & 5.47 & 0.103 \\
\hline 94 & 1995/01/10/07:55 & 23.68 & 121.43 & 3.81 & 5.1 & 4.8 & 5.12 & 0.062 \\
\hline 95 & $1995 / 02 / 23 / 05: 19$ & 24.20 & 121.69 & 21.69 & 5.8 & 6.2 & 5.77 & 2.45 \\
\hline 96 & $1995 / 04 / 03 / 11: 54$ & 23.94 & 122.43 & 14.55 & 5.7 & 5.5 & 5.88 & 0.365 \\
\hline 97 & $1995 / 04 / 03 / 22: 33$ & 23.95 & 122.32 & 3.94 & 5.3 & 5.0 & 5.35 & 0.12 \\
\hline 98 & $1995 / 04 / 09 / 04: 44$ & 21.83 & 121.03 & 15.70 & 5.2 & 4.7 & 5.33 & 0.08 \\
\hline 99 & 1995/05/27/18:11 & 23.01 & 121.46 & 19.73 & 5.2 & 5.6 & 5.26 & 0.402 \\
\hline 100 & 1995/06/25/06:59 & 24.61 & 121.67 & 39.88 & 5.8 & 5.7 & 6.50 & 1.022 \\
\hline 101 & $1996 / 03 / 05 / 14: 52$ & 23.93 & 122.36 & 6.00 & 6.1 & 6.4 & 6.40 & 3.59 \\
\hline 102 & $1996 / 03 / 05 / 17: 32$ & 23.90 & 122.30 & 10.81 & 5.6 & 5.6 & 5.96 & 0.733 \\
\hline 103 & $1996 / 03 / 29 / 03: 28$ & 23.97 & 122.33 & 5.79 & 5.4 & 5.5 & 5.64 & 0.52 \\
\hline 104 & 1996/08/10/06:23 & 23.89 & 122.65 & 5.65 & 5.3 & 5.3 & 5.76 & 0.342 \\
\hline 105 & $1996 / 09 / 05 / 23: 42$ & 22.00 & 121.37 & 14.76 & 6.4 & 6.6 & 7.07 & 19.1 \\
\hline
\end{tabular}


Table 1. (Continued)

\begin{tabular}{ccccccccc}
\hline No. & Time & Latitude & Longitude & Depth & & & & \\
& & $\left({ }^{\mathbf{0}}\right)$ & $\left({ }^{\mathbf{0}} \mathbf{E}\right)$ & $(\mathbf{k m})$ & $\mathbf{m}_{\mathbf{b}}$ & $\mathbf{M}_{\mathbf{s}}$ & $\mathbf{M}_{\mathbf{L}}$ & $\mathbf{M}_{\mathbf{o}}$ \\
\hline 106 & $1996 / 09 / 06 / 11: 34$ & 21.69 & 121.32 & 19.90 & 5.5 & 4.9 & 5.59 & 0.138 \\
107 & $1996 / 11 / 26 / 08: 22$ & 24.16 & 121.70 & 26.18 & 5.1 & 4.8 & 5.35 & 0.077 \\
108 & $1997 / 01 / 05 / 10: 34$ & 24.62 & 122.53 & 1.13 & 5.3 & 4.9 & 5.78 & 0.074 \\
109 & $1997 / 05 / 03 / 02: 46$ & 22.54 & 121.40 & 3.64 & 5.1 & 5.0 & 5.27 & 0.035 \\
110 & $1997 / 06 / 22 / 09: 36$ & 22.17 & 121.38 & 1.83 & 5.2 & 4.9 & 5.40 & 0.076 \\
111 & $1997 / 07 / 04 / 18: 37$ & 23.06 & 120.79 & 5.16 & 4.9 & 4.7 & 5.18 & 0.05 \\
112 & $1997 / 08 / 24 / 12: 17$ & 21.64 & 120.20 & 41.53 & 5.3 & 4.6 & 5.25 & 0.146 \\
113 & $1997 / 08 / 24 / 18: 58$ & 21.63 & 120.27 & 45.62 & 5.3 & 5.0 & 5.35 & 0.105 \\
114 & $1998 / 01 / 18 / 19: 56$ & 22.73 & 121.09 & 3.28 & 4.8 & 4.7 & 5.07 & 0.073 \\
115 & $1998 / 07 / 17 / 04: 51$ & 23.50 & 120.66 & 2.80 & 5.5 & 5.4 & 6.20 & 0.431 \\
116 & $1998 / 07 / 24 / 18: 44$ & 21.63 & 121.84 & 6.67 & 5.6 & 5.9 & 5.93 & 1.73 \\
117 & $1998 / 09 / 13 / 05: 34$ & 24.24 & 123.01 & 28.11 & 5.2 & 4.6 & 5.82 & 0.122 \\
118 & $1998 / 11 / 17 / 22: 27$ & 22.83 & 120.79 & 16.49 & 5.2 & 5.1 & 5.51 & 0.113 \\
119 & $1999 / 02 / 22 / 13: 49$ & 23.98 & 122.65 & 4.21 & 5.4 & 5.6 & 5.90 & 0.805 \\
120 & $1999 / 09 / 10 / 14: 18$ & 22.44 & 121.82 & 5.19 & 5.4 & 4.8 & 5.41 & 0.144 \\
121 & $1999 / 09 / 20 / 17: 47$ & 23.85 & 120.82 & 8.00 & 6.5 & 7.7 & 7.30 & 338 \\
122 & $1999 / 09 / 20 / 21: 46$ & 23.58 & 120.86 & 8.57 & 5.8 & 6.5 & 6.59 & 4.83 \\
123 & $1999 / 09 / 22 / 00: 14$ & 23.83 & 121.05 & 15.59 & 6.2 & 6.4 & 6.80 & 5.03 \\
124 & $1999 / 09 / 22 / 00: 49$ & 23.76 & 121.03 & 17.38 & 5.9 & 5.9 & 6.20 & 0.631 \\
125 & $1999 / 09 / 22 / 12: 17$ & 23.74 & 120.98 & 24.02 & 5.1 & 4.9 & 6.00 & 0.093 \\
126 & $1999 / 09 / 23 / 12: 44$ & 23.93 & 121.09 & 18.35 & 5.3 & 4.8 & 5.63 & 0.088 \\
127 & $1999 / 09 / 25 / 08: 43$ & 23.69 & 120.95 & 7.12 & 5.2 & 4.8 & 5.09 & 0.051 \\
128 & $1999 / 09 / 25 / 23: 52$ & 23.85 & 121.00 & 12.06 & 6.2 & 6.4 & 6.80 & 6.01 \\
129 & $1999 / 10 / 02 / 17: 14$ & 23.96 & 122.50 & 6.59 & 5.0 & 4.8 & 5.30 & 0.061 \\
130 & $1999 / 10 / 22 / 02: 18$ & 23.52 & 120.42 & 16.59 & 5.7 & 5.6 & 6.40 & 0.695 \\
131 & $1999 / 10 / 22 / 03: 10$ & 23.53 & 120.43 & 16.74 & 5.2 & 5.3 & 6.00 & 0.251 \\
132 & $1999 / 10 / 30 / 08: 27$ & 24.02 & 121.32 & 14.36 & 5.1 & 4.6 & 5.15 & 0.133 \\
\hline & $1999 / 11 / 01 / 17: 53$ & 23.36 & 121.73 & 31.33 & 6.1 & 6.1 & 6.90 & 3.29 \\
\hline
\end{tabular}


Table 1. (Continued)

\begin{tabular}{ccccccccc}
\hline No. & Time & Latitude & Longitude & $\begin{array}{c}\text { Depth } \\
\mathbf{(} \mathbf{N})\end{array}$ & $\left.\mathbf{m}_{\mathbf{b}} \mathbf{E}\right)$ & $\mathbf{M}_{\mathbf{s}}$ & $\mathbf{M}_{\mathbf{L}}$ & $\mathbf{M}_{\mathbf{o}}$ \\
\hline 134 & $2000 / 02 / 15 / 21: 33$ & 23.32 & 120.74 & 14.71 & 5.3 & 4.9 & 5.59 & 0.085 \\
135 & $2000 / 05 / 17 / 03: 25$ & 24.19 & 121.10 & 9.74 & 5.4 & 5.3 & 5.59 & 0.161 \\
136 & $2000 / 06 / 10 / 18: 23$ & 23.90 & 121.11 & 16.21 & 6.2 & 6.2 & 6.70 & 5.35 \\
137 & $2000 / 06 / 19 / 21: 56$ & 23.92 & 121.09 & 27.02 & 5.0 & 4.6 & 5.18 & 0.092 \\
138 & $2000 / 07 / 14 / 00: 07$ & 24.05 & 121.73 & 7.19 & 5.2 & 5.0 & 5.70 & 0.135 \\
139 & $2000 / 07 / 28 / 20: 28$ & 23.41 & 120.93 & 7.35 & 5.6 & 5.6 & 6.10 & 0.345 \\
140 & $2000 / 08 / 23 / 00: 49$ & 23.64 & 121.63 & 27.48 & 5.2 & 4.9 & 5.57 & 0.113 \\
141 & $2000 / 09 / 10 / 08: 54$ & 24.09 & 121.58 & 17.74 & 5.6 & 5.6 & 6.20 & 0.583 \\
142 & $2000 / 09 / 16 / 23: 04$ & 23.92 & 122.50 & 15.10 & 5.2 & 4.8 & 5.30 & 0.089 \\
143 & $2000 / 12 / 12 / 20: 32$ & 23.97 & 122.68 & 19.43 & 5.1 & 5.3 & 5.29 & 0.157 \\
144 & $2001 / 03 / 01 / 16: 37$ & 23.84 & 121.00 & 10.93 & 5.3 & 4.9 & 5.80 & 0.089 \\
145 & $2001 / 06 / 14 / 02: 35$ & 24.42 & 121.93 & 17.29 & 5.7 & 5.6 & 6.30 & 0.78 \\
146 & $2001 / 06 / 19 / 05: 16$ & 23.18 & 121.08 & 6.58 & 5.0 & 4.8 & 5.41 & 0.119 \\
147 & $2001 / 06 / 19 / 05: 43$ & 23.20 & 121.10 & 11.70 & 5.1 & 4.7 & 5.22 & 0.058 \\
148 & $2001 / 11 / 10 / 00: 37$ & 24.88 & 122.71 & 5.00 & 5.0 & 4.8 & 4.81 & 0.049 \\
149 & $2001 / 12 / 18 / 04: 02$ & 23.87 & 122.65 & 12.00 & 6.3 & 7.3 & 6.70 & 20.8 \\
150 & $2001 / 12 / 22 / 21: 40$ & 24.12 & 122.91 & 8.73 & 5.0 & 4.8 & 5.39 & 0.061 \\
151 & $2001 / 12 / 28 / 00: 41$ & 23.99 & 122.90 & 9.46 & 5.2 & 4.9 & 5.49 & 0.057 \\
152 & $2002 / 02 / 12 / 03: 27$ & 23.74 & 121.72 & 29.98 & 5.8 & 5.4 & 6.20 & 0.379 \\
153 & $2002 / 03 / 31 / 06: 52$ & 24.14 & 122.19 & 13.81 & 6.4 & 7.4 & 6.80 & 54.5 \\
154 & $2002 / 04 / 03 / 18: 06$ & 24.32 & 121.87 & 12.87 & 5.0 & 4.9 & 5.27 & 0.1 \\
155 & $2002 / 04 / 28 / 13: 23$ & 24.13 & 122.87 & 9.18 & 5.3 & 4.6 & 5.54 & 0.07 \\
156 & $2002 / 05 / 15 / 03: 46$ & 24.65 & 121.87 & 8.52 & 5.5 & 6.2 & 6.20 & 1.91 \\
157 & $2002 / 05 / 28 / 16: 45$ & 23.91 & 122.40 & 15.23 & 5.8 & 5.9 & 6.20 & 1.49 \\
158 & $2002 / 06 / 13 / 20: 40$ & 24.78 & 122.13 & 8.14 & 4.9 & 4.7 & 5.03 & 0.066 \\
159 & $2002 / 07 / 11 / 07: 36$ & 23.94 & 122.41 & 14.22 & 5.6 & 5.6 & 5.79 & 0.652 \\
160 & $2002 / 07 / 13 / 12: 07$ & 23.80 & 122.68 & 6.26 & 5.0 & 4.4 & 5.14 & 0.029 \\
161 & $2002 / 08 / 28 / 17: 05$ & 22.26 & 121.37 & 12.03 & 5.7 & 5.1 & 6.03 & 0.255 \\
\hline & & & & & & &
\end{tabular}


Table 1. (Continued)

\begin{tabular}{|c|c|c|c|c|c|c|c|c|}
\hline No. & Time & $\begin{array}{c}\text { Latitude } \\
\left({ }^{0} \mathrm{~N}\right)\end{array}$ & $\begin{array}{c}\text { Longitude } \\
\left({ }^{\circ} \mathrm{E}\right)\end{array}$ & $\begin{array}{c}\text { Depth } \\
(\mathrm{km})\end{array}$ & $\mathbf{m}_{b}$ & $\mathbf{M}_{\mathbf{s}}$ & $\mathbf{M}_{\mathbf{L}}$ & $\mathbf{M}_{\mathbf{0}}$ \\
\hline 162 & 2002/09/01/05:56 & 23.92 & 122.43 & 8.81 & 5.5 & 5.7 & 5.46 & 0.183 \\
\hline 163 & 2002/09/01/07:07 & 23.97 & 122.37 & 15.56 & 5.3 & 5.4 & 5.48 & 0.112 \\
\hline 164 & 2002/09/15/01:06 & 23.92 & 122.53 & 11.03 & 5.0 & 4.7 & 5.17 & 0.058 \\
\hline 165 & 2002/12/21/06:09 & 21.53 & 121.37 & 12.29 & 5.1 & 5.0 & 5.01 & 0.056 \\
\hline 166 & $2003 / 05 / 15 / 01: 17$ & 25.06 & 122.52 & 17.58 & 4.8 & 4.9 & 5.21 & 0.054 \\
\hline 167 & 2003/06/09/01:52 & 24.37 & 122.02 & 23.22 & 5.3 & 5.5 & 5.72 & 0.642 \\
\hline 168 & $2003 / 06 / 10 / 08: 40$ & 23.50 & 121.70 & 32.31 & 5.8 & 5.7 & 6.48 & 1.041 \\
\hline 169 & $2003 / 07 / 30 / 18: 36$ & 23.92 & 122.46 & 12.34 & 4.9 & 4.6 & 5.15 & 0.078 \\
\hline 170 & $2003 / 12 / 10 / 04: 38$ & 23.07 & 121.40 & 17.73 & 6.0 & 6.7 & 6.42 & 20.03 \\
\hline 171 & 2003/12/11/00:01 & 22.79 & 121.39 & 33.58 & 5.1 & 5.1 & 5.39 & 0.179 \\
\hline 172 & $2003 / 12 / 17 / 16: 27$ & 22.61 & 121.31 & 32.20 & 5.2 & 5.1 & 5.43 & 0.121 \\
\hline 173 & 2004/01/01/03:15 & 23.34 & 121.71 & 24.88 & 5.1 & 4.8 & 5.35 & 0.069 \\
\hline 174 & $2004 / 02 / 04 / 03: 24$ & 23.38 & 122.15 & 17.36 & 5.4 & 5.0 & 5.64 & 0.138 \\
\hline 175 & $2004 / 05 / 01 / 07: 56$ & 24.08 & 121.53 & 21.55 & 5.2 & 5.1 & 5.25 & 0.073 \\
\hline 176 & 2004/05/08/08:02 & 21.93 & 121.64 & 6.61 & 5.7 & 5.4 & 5.59 & 0.25 \\
\hline 177 & 2004/05/16/06:04 & 23.05 & 121.98 & 12.85 & 5.3 & 5.0 & 5.72 & 0.262 \\
\hline 178 & 2004/05/19/07:04 & 22.71 & 121.37 & 27.08 & 5.8 & 6.1 & 6.03 & 2.57 \\
\hline 179 & $2004 / 07 / 06 / 07: 32$ & 24.90 & 122.27 & 5.96 & 4.8 & 4.7 & 5.22 & 0.081 \\
\hline 180 & $2004 / 11 / 08 / 15: 55$ & 23.79 & 122.76 & 10.00 & 5.9 & 6.1 & 6.58 & 3.202 \\
\hline 181 & $2004 / 11 / 08 / 19: 38$ & 23.93 & 122.51 & 15.74 & 5.3 & 5.2 & 5.50 & 0.069 \\
\hline 182 & $2004 / 11 / 10 / 14: 48$ & 23.97 & 122.42 & 14.87 & 5.2 & 4.8 & 5.15 & 0.044 \\
\hline 183 & $2004 / 11 / 11 / 02: 16$ & 24.31 & 122.16 & 27.26 & 5.7 & 5.5 & 6.09 & 0.359 \\
\hline 184 & $2004 / 12 / 16 / 00: 10$ & 23.95 & 122.41 & 8.56 & 5.0 & 5.1 & 5.13 & 0.049 \\
\hline 185 & $2005 / 02 / 18 / 20: 18$ & 23.34 & 121.67 & 15.28 & 5.6 & 5.2 & 5.60 & 0.154 \\
\hline 186 & 2005/03/05/19:06 & 24.65 & 121.80 & 6.95 & 5.7 & 5.6 & 5.96 & 0.528 \\
\hline 187 & $2005 / 04 / 30 / 14: 48$ & 24.04 & 121.62 & 8.45 & 5.2 & 4.9 & 5.62 & 0.103 \\
\hline 188 & $2005 / 06 / 07 / 16: 45$ & 23.99 & 121.74 & 2.09 & 5.1 & 4.6 & 5.16 & 0.036 \\
\hline 189 & 2005/07/20/13:06 & 24.75 & 122.25 & 7.60 & 5.1 & 4.9 & 5.22 & 0.093 \\
\hline
\end{tabular}


Table 1. (Continued)

\begin{tabular}{ccccccccc}
\hline No. & Time & $\begin{array}{c}\text { Latitude } \\
\left({ }^{0} \mathbf{N}\right)\end{array}$ & $\begin{array}{c}\text { Longitude } \\
\left({ }^{\mathbf{0}} \mathbf{E}\right)\end{array}$ & $\begin{array}{c}\text { Depth } \\
(\mathbf{k m})\end{array}$ & $\mathbf{m}_{\mathbf{b}}$ & $\mathbf{M}_{\mathbf{s}}$ & $\mathbf{M}_{\mathbf{L}}$ & $\mathbf{M}_{\mathbf{0}}$ \\
\hline 190 & $2005 / 09 / 06 / 01: 16$ & 23.96 & 122.28 & 16.76 & 5.8 & 6.1 & 6.00 & 0.552 \\
191 & $2005 / 11 / 16 / 13: 39$ & 23.93 & 122.50 & 20.87 & 4.9 & 4.2 & 5.08 & 0.014 \\
192 & $2006 / 01 / 22 / 07: 07$ & 23.96 & 122.32 & 5.83 & 5.1 & 4.8 & 4.94 & 0.028 \\
193 & $2006 / 01 / 23 / 04: 18$ & 24.01 & 122.29 & 17.62 & 5.0 & 4.5 & 4.86 & 0.021 \\
194 & $2006 / 04 / 01 / 10: 02$ & 22.88 & 121.08 & 7.20 & 6.0 & 6.2 & 6.23 & 1.992 \\
195 & $2006 / 04 / 15 / 22: 40$ & 22.86 & 121.30 & 17.90 & 5.5 & 5.9 & 6.04 & 0.874 \\
196 & $2006 / 04 / 28 / 09: 05$ & 23.99 & 121.61 & 9.78 & 5.1 & 5.6 & 5.21 & 0.084 \\
197 & $2006 / 05 / 07 / 01: 53$ & 21.59 & 120.73 & 42.46 & 5.4 & 5.4 & 5.23 & 0.116 \\
198 & $2006 / 06 / 05 / 00: 39$ & 21.38 & 122.05 & 19.98 & 4.9 & 4.5 & 5.02 & 0.04 \\
199 & $2006 / 07 / 28 / 07: 40$ & 23.97 & 122.66 & 27.97 & 5.7 & 5.9 & 6.02 & 0.76 \\
200 & $2006 / 08 / 11 / 23: 51$ & 22.54 & 121.40 & 25.40 & 5.4 & 5.1 & 5.39 & 0.074 \\
201 & $2006 / 10 / 12 / 14: 46$ & 23.96 & 122.65 & 25.30 & 5.7 & 5.7 & 5.80 & 0.532 \\
\hline
\end{tabular}

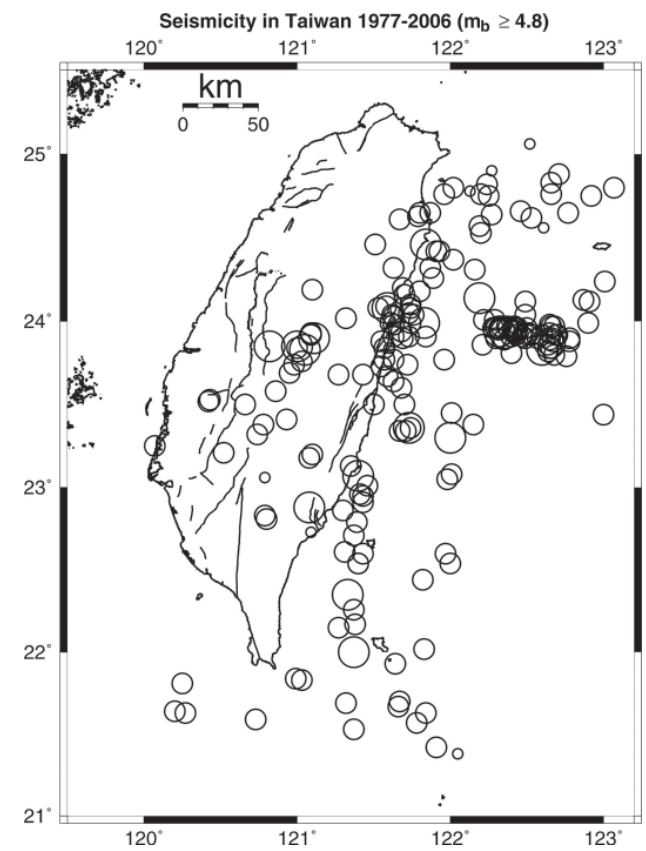

Fig. 1. The epicentral distribution of earthquakes shown in Table 1. 


\section{RESULTS}

According to the source parameters of these 201 events, the plots of $\log \left(\mathrm{M}_{\mathrm{o}}\right)$ versus $\mathrm{M}_{\mathrm{s}}$, $\log \left(M_{o}\right)$ versus $m_{b}$, and $M_{s}$ versus $m_{b}$ are shown in Figs. 2 - 4, respectively. Obviously, a linear relationship exists between any two parameters. Hence, the $\log \left(M_{o}\right)-M s, \log \left(M_{o}\right)-m_{b}$, and $\mathrm{M}_{\mathrm{s}}-\mathrm{m}_{\mathrm{b}}$ relationships are evaluated through the least- square method. The resulting formulas are:

$$
\begin{aligned}
& \log \left(\mathrm{M}_{\mathrm{o}}\right)=(1.07 \pm 0.04) \mathrm{M}_{\mathrm{s}}+(18.72 \pm 0.20) \\
& \log \left(\mathrm{M}_{\mathrm{o}}\right)=(1.73 \pm 0.09) \mathrm{m}_{\mathrm{b}}+(15.09 \pm 0.52) \\
& \mathrm{M}_{\mathrm{s}}=(1.46 \pm 0.08) \mathrm{m}_{\mathrm{b}}-(2.52 \pm 0.43)
\end{aligned}
$$

The related regression lines are depicted with a solid line, respectively, in Figs. 2 - 4. The number next to each circle presents the event number listed in Table 1. In Fig. 2, the $\mathbf{M}_{o}-\mathrm{M}_{\mathrm{s}}$ relation fits the given data comparatively well. In comparison of Fig. 2 with Fig. 3, it can be found that for the given data, there is more scattering in the plot of $M_{o}$ versus $M_{s}$ than that of $M_{o}$ versus $m_{b}$, especially for $M_{s} \leq 5.6$. This might be due to the fact that the number of readings used to estimate the magnitude is usually smaller for $M_{s}$ than for $m_{b}$; therefore, a higher uncertainty for $\mathrm{M}_{\mathrm{s}}$ than $\mathrm{m}_{\mathrm{b}}$ is unavoidable.

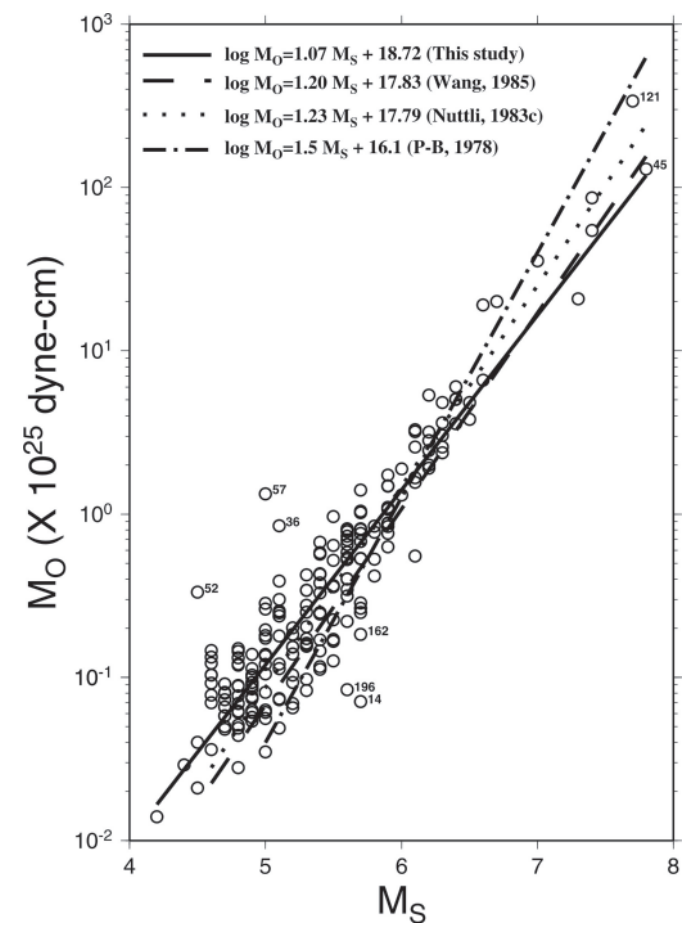

Fig. 2. The plot of $\log \left(\mathrm{M}_{\mathrm{o}}\right)$ versus $\mathrm{M}_{\mathrm{s}}$ and related regression lines. The number next to the dot denotes the event number shown in Table 1. The solid line is deduced by this study. The dashed line is given by Wang (1985). The dotted line is given by Nuttli (1985). The dotted-dashed line is taken from Purcaru and Berckhemer (denoted by P-B 1978). 

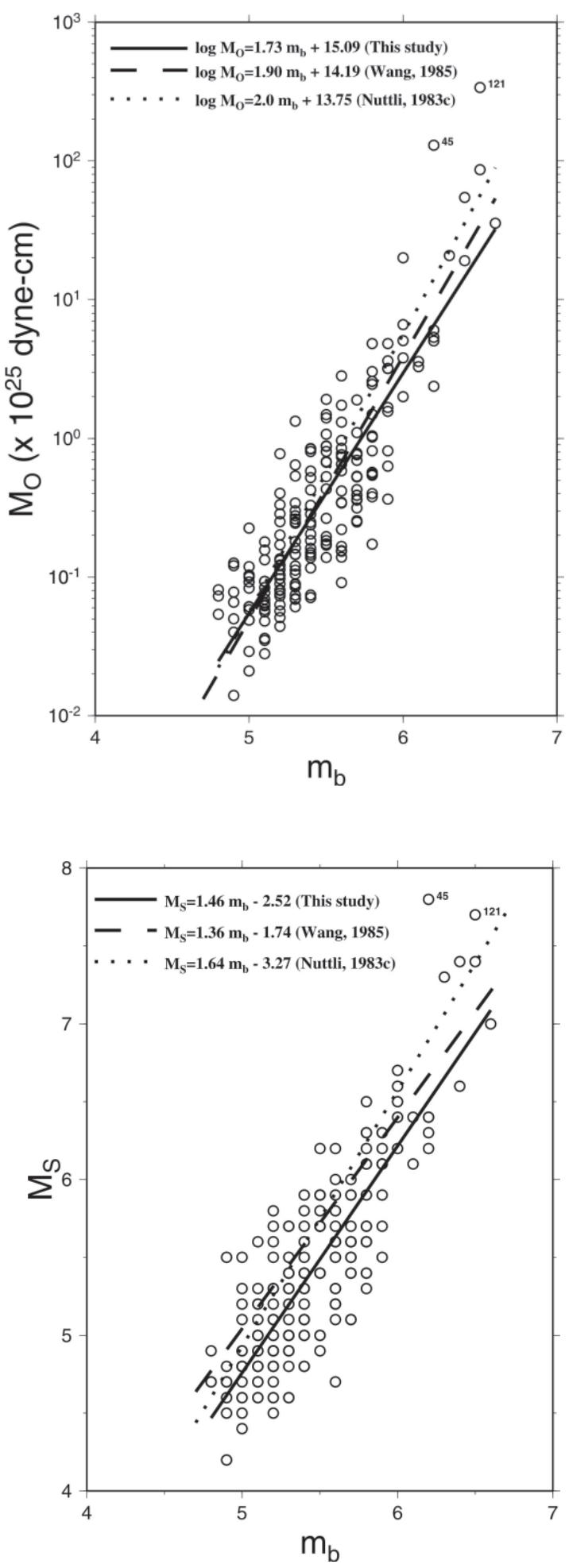

Fig. 3. The plot of $\log \left(\mathrm{M}_{\mathrm{o}}\right)$ versus $\mathrm{m}_{\mathrm{b}}$ and related regression lines. The associated symbols are the same as in Fig. 2.

Fig. 4. The plot of $\mathrm{M}_{\mathrm{s}}$ versus $\mathrm{m}_{\mathrm{b}}$ and the related regression lines. The associated symbols are the same as in Fig. 2. 
For 125 events with local magnitude, the plots of $\log \left(\mathrm{M}_{\mathrm{o}}\right)$ versus $\mathrm{M}_{\mathrm{L}}, \mathrm{m}_{\mathrm{b}}$ versus $\mathrm{M}_{\mathrm{L}}$, and $M_{s}$ versus $M_{L}$ are shown in Figs. 5 - 7, respectively. Obviously, a linear relationship exists between any two parameters. Hence, the $\log \left(\mathrm{M}_{\mathrm{o}}\right)-\mathrm{M}_{\mathrm{L}}, \mathrm{m}_{\mathrm{b}}-\mathrm{M}_{\mathrm{L}}$, and $\mathrm{M}_{\mathrm{s}}-\mathrm{M}_{\mathrm{L}}$ relationships evaluated through the least-square method are:

$$
\begin{aligned}
& \log \left(\mathrm{M}_{\mathrm{o}}\right)=(1.27 \pm 0.06) \mathrm{M}_{\mathrm{L}}+(17.23 \pm 0.35) \\
& \mathrm{m}_{\mathrm{b}}=(0.66 \pm 0.03) \mathrm{M}_{\mathrm{L}}+(1.69 \pm 0.17) \\
& \mathrm{M}_{\mathrm{s}}=(1.03 \pm 0.06) \mathrm{M}_{\mathrm{L}}-(0.53 \pm 0.36)
\end{aligned}
$$

The related regression lines are depicted with a solid line, respectively, in Figs. 5 - 7.

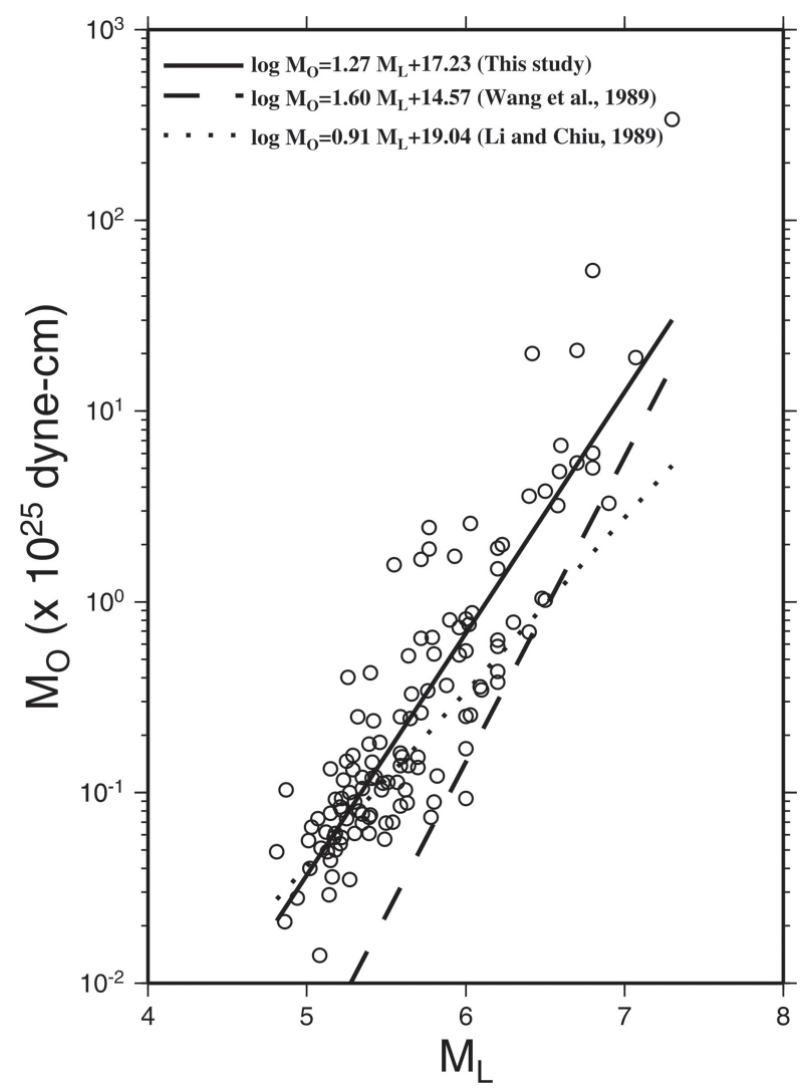

Fig. 5. The plot of $\log \left(\mathrm{M}_{\mathrm{o}}\right)$ versus $\mathrm{M}_{\mathrm{L}}$ and related regression lines. The solid line is deduced by this study. The dashed line is given by Wang et al. (1989). The dotted line is given by $\mathrm{Li}$ and Chiu (1989). 


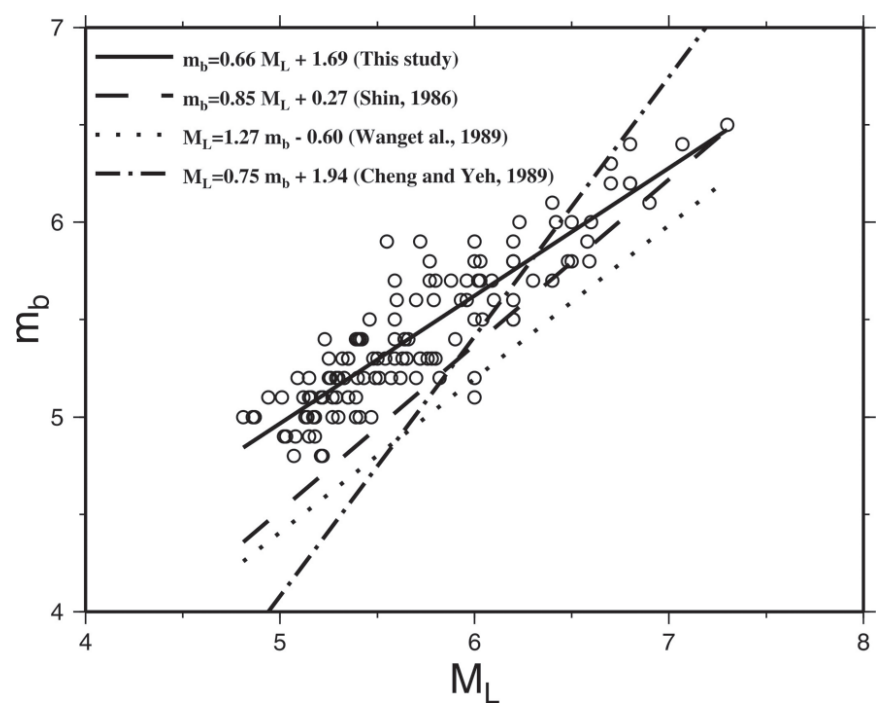

Fig. 6. The plot of $\mathrm{m}_{\mathrm{b}}$ versus $\mathrm{M}_{\mathrm{L}}$ and the related regression lines. The solid line is deduced by this study. The dashed line is given by Shin (1986). The dotted line is given by Wang et al. (1989). The dotted-dashed line is taken from Cheng and Yeh (1989).

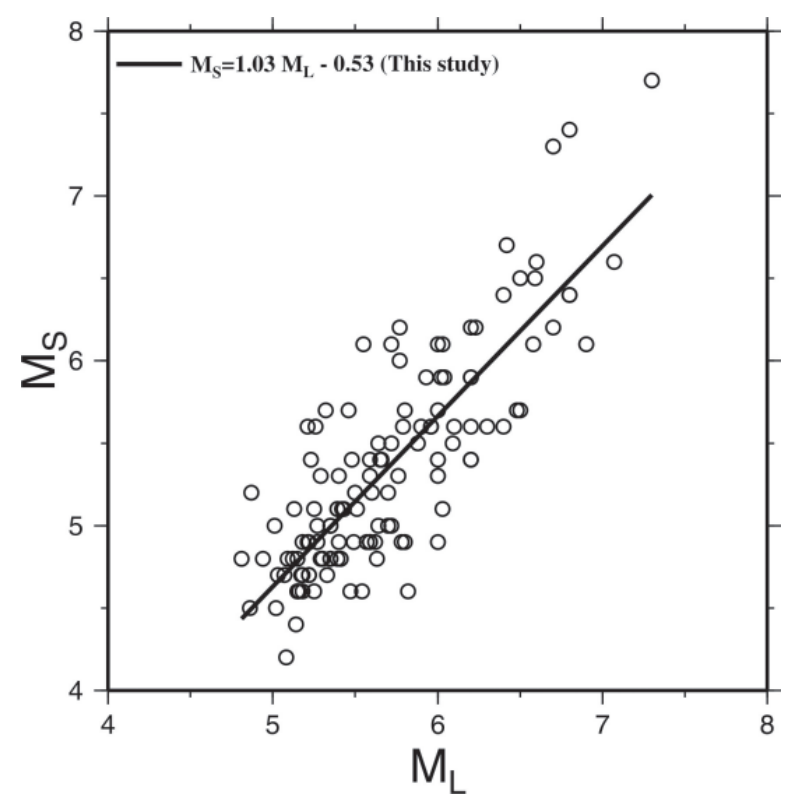

Fig. 7. The plot of $\mathrm{M}_{\mathrm{s}}$ versus $\mathrm{M}_{\mathrm{L}}$ and the related regression line. The solid line is deduced by this study. 


\section{DISCUSSION}

Figure 2 shows that the data points related to three events (event No. 36, 52, and 57) with $M_{s} \leq 5$ are above the cluster of data points and deviate greatly from Eq. (1). This deviation might be due to over-estimates of $\mathrm{M}_{\mathrm{o}}$ or under-estimates of $\mathrm{M}_{\mathrm{s}}$ for the three events. Meanwhile, the data points related to three events (event No. 14, 162, and 196) with $\mathrm{M}_{\mathrm{s}}<6$ are below the cluster of data points and deviate also from Eq. (1). This might be due to under-estimates of $\mathbf{M}_{\mathrm{o}}$ or over-estimates of $\mathrm{M}_{\mathrm{s}}$ for those events.

Figures 2 - 4 show that the data point (event No. 45), with $\mathrm{M}_{\mathrm{s}}=7.8, \mathrm{~m}_{\mathrm{b}}=6.2$, and $\mathrm{M}_{\mathrm{o}}=$ $1.30 \times 10^{27}$ dyne-cm, related to the 1986 Offshore Hualien earthquake (Chen and Wang 1988) and that the data point (event No. 121), with $\mathrm{M}_{\mathrm{s}}=7.7, \mathrm{~m}_{\mathrm{b}}=6.5$, and $\mathrm{M}_{\mathrm{o}}=3.38 \times 10^{27}$ dyne-cm, associated with the 1999 Chi-Chi earthquake (Ma et al. 1999) depart from Eq. (2). Two possible reasons can cause the observations. The first one is saturation of amplitudes around 1 second, which are used for determining the $\mathrm{m}_{\mathrm{b}}$ scale, usually contributes to under-estimation of $\mathrm{m}_{\mathrm{b}}$ for large earthquakes. The second one is the existence of stronger low-frequency spectral amplitudes than high-frequency ones for the two events. For the 1999 earthquake, there are numerous observations (Hwang et al. 2001; Huang and Wang 2002; Wang et al. 2002; Wang 2006a, b) to confirm this possibility, while for the 1986 event information is insufficient. In addition, $\mathbf{M}_{\mathrm{o}}$ is higher for the 1999 Chi-Chi earthquake than for the 1986 Offshore Hualien earthquake, yet the opposite is true for $\mathrm{M}_{\mathrm{s}}$. This indicates that low-frequency spectral amplitudes are higher for the former than for the latter.

As mentioned above, Wang (1985) first estimated the relationships of $M_{o}$ versus $M_{s}, M_{o}$ versus $m_{b}$, and $M_{s}$ versus $m_{b}$ for 16 earthquakes in the Taiwan region. The results are:

$$
\begin{aligned}
& \log \left(\mathrm{M}_{\mathrm{o}}\right)=1.20 \mathrm{M}_{\mathrm{s}}+17.83, \\
& \log \left(\mathrm{M}_{\mathrm{o}}\right)=1.90 \mathrm{~m}_{\mathrm{b}}+14.19 \\
& \mathrm{M}_{\mathrm{s}}=1.36 \mathrm{~m}_{\mathrm{b}}-1.74
\end{aligned}
$$

Equations (7) - (9) are depicted with dashed lines, respectively, in Figs. 2 - 4. Obviously, the dashed lines are close to the solid lines and, thus, Eqs. (7) - (9) can describe the data points well. This indicates that Eqs. (7) - (9) are reliable even though they were inferred from a small number of data.

For global earthquakes, the $M_{o}-M_{s}$ and $M_{o}-m_{b}$, and $M_{s}-m_{b}$ relationships inferred by Nuttli (1985) are, respectively:

$$
\begin{aligned}
& \log \left(\mathrm{M}_{\mathrm{o}}\right)=1.23 \mathrm{M}_{\mathrm{s}}+17.79 \quad\left(6.2 \leq \mathrm{M}_{\mathrm{s}} \leq 7.8\right), \\
& \log \left(\mathrm{M}_{\mathrm{o}}\right)=2.00 \mathrm{~m}_{\mathrm{b}}+13.75 \quad\left(4.4 \leq \mathrm{M}_{\mathrm{s}} \leq 6.9\right), \\
& \mathrm{M}_{\mathrm{s}}=1.64 \mathrm{~m}_{\mathrm{b}}-3.27 .
\end{aligned}
$$

Equations (10)- (12) are depicted with dotted lines, respectively, in Figs. 2 - 4. The dotted lines 
do not depart from the solid lines too much. This leads to the same conclusion stated by Wang (1985) that $\mathrm{M}_{\mathrm{o}}-\mathrm{M}_{\mathrm{s}}, \mathrm{M}_{\mathrm{o}}-\mathrm{m}_{\mathrm{b}}$, and $\mathrm{M}_{\mathrm{s}}-\mathrm{m}_{\mathrm{b}}$ relationships for earthquakes in the Taiwan region have good agreement with the average relations for plate-margin earthquakes obtained by Nuttli (1985).

The empirical $M_{o}-M_{s}$ relationship inferred by Purcaru and Berckhember (1978) for $5 \leq$ $\mathrm{M}_{\mathrm{s}} \leq 7.5$ is:

$$
\log \left(\mathrm{M}_{\mathrm{o}}\right)=1.5 \mathrm{M}_{\mathrm{s}}+16.1
$$

which is depicted with a dotted-dashed line in Fig. 2. Obviously, Eq. (13) departs from Eq. (1) and cannot describe the data points well.

A conjecture based on the above-mentioned results and Nuttli's proposition (1985) that spectral scaling and source parameter studies can be carried out for individual earthquake source zones might be figured out. The conjecture is that the tectonics of the Taiwan region behave like the average property of the circum-Pacific oceanic plate margin. This conjecture can explain the agreement of relationships in this region with Nuttli's results (1985). Moreover, the earthquake magnitude has a close relation with seismic energy. Thus, the seismic momentmagnitude relation will reflect the accumulation of energy due to regional tectonic activities. Consequently, the above- mentioned agreement displays that the preparatory process of earthquake energy and source properties of the Taiwan region are the average ones of the circumPacific oceanic seismic belt.

Equation (1) and Fig. 2 show $\log \left(\mathbf{M}_{\mathrm{o}}\right) \sim \mathrm{M}_{\mathrm{s}}$ as proposed by Aki (1972) and Koyama (1994) for $\mathrm{M}_{\mathrm{s}}<7$ and by Ekstrom and Dziewonski (1988) for $\mathrm{M}_{\mathrm{s}}<5.8$ rather than $\log \left(\mathrm{M}_{\mathrm{o}}\right) \sim 1.5 \mathrm{M}_{\mathrm{s}}$ as suggested by Kanamori and Anderson (1975) for large earthquakes. Nuttli (1985) almost obtained a similar correlation from Eq. (10), yet not from Eq. (13) by Purcaru and Berckhember (1978). The definition of seismic moment is $\mathrm{M}_{o}=\mu \mathrm{DLW}$, where $\mu, \mathrm{D}, \mathrm{L}$, and $\mathrm{W}$ are, respectively, the rigidity of materials in the source region, the average displacement on a fault plane, the fault length, and the fault depth. D is usually proportional to L rather than W (cf. Wang and Ou 1988). Kanamori and Anderson (1975) assumed $\mathrm{W} \sim \mathrm{L}$, thus leading to $\mathrm{M}_{\mathrm{o}} \sim \mathrm{L}^{3}$ or $\log \left(\mathrm{M}_{\mathrm{o}}\right) \sim 3 \log (\mathrm{L})$. Let $\tau$ and $\mathrm{v}$ be the rise time and rupture velocity of an earthquake, respectively. For most large earthquakes, they proposed $\mathrm{M}_{\mathrm{s}} \sim 2 \log (\mathrm{L})$ when $\tau<\mathrm{T}_{\mathrm{o}} / \pi$ and $(\mathrm{L} / \mathrm{v})>\mathrm{T}_{\mathrm{o}} / \pi$, where $\mathrm{T}_{\mathrm{o}}=20 \mathrm{sec}$ of wave motions. Hence, the two correlations result in $\log \left(\mathrm{M}_{\mathrm{o}}\right) \sim 1.5 \mathrm{M}_{\mathrm{s}}$. This contrasts with our observation. On the other hand, for moderate earthquakes Kanamori and Anderson (1975) also suggested $\mathrm{M}_{\mathrm{s}} \sim 3 \log (\mathrm{L})$ when $\tau<\mathrm{T}_{\mathrm{o}} / \pi$ and $(\mathrm{L} / \mathrm{v})<\mathrm{T}_{\mathrm{o}} / \pi$. This leads to $\log \left(\mathrm{M}_{\mathrm{o}}\right) \sim \mathrm{M}_{\mathrm{s}}$. Based on Haskell's model (Haskell 1964), Geller (1976) obtained $\log \left(\mathrm{M}_{\mathrm{o}}\right) \sim \mathrm{M}_{\mathrm{s}}$ when $\mathrm{M}_{\mathrm{s}}<6.76$. His theoretical result can interpret our observation.

Equations (2) and (11) both suggest $\log \left(\mathrm{M}_{\mathrm{o}}\right) \sim 2 \mathrm{~m}_{\mathrm{b}}$. This observed correction is inconsistent with the theoretical correlation: $\log \left(\mathrm{M}_{\mathrm{o}}\right) \sim 2.5 \mathrm{~m}_{\mathrm{b}}$ proposed by Koyama (1994) for three kinds of distributions of peak and trough amplitudes. Hence, his proposition is questionable and needs further study for exploring the theoretical relationship between $\mathrm{M}_{\mathrm{o}}$ and $\mathrm{m}_{\mathrm{b}}$.

Equation (3) and Fig. 4 show $\mathrm{M}_{\mathrm{s}} \sim 1.5 \mathrm{~m}_{\mathrm{b}}$. Nuttli (1985) almost obtained a similar correlation from Eq. (12). According to Haskell's source model, Geller (1976) obtained: (1) $\mathrm{M}_{\mathrm{s}} \sim 1.5 \mathrm{~m}_{\mathrm{b}}$ when $2.86<\mathrm{M}_{\mathrm{s}}<4.90$; (2) $\mathrm{M}_{\mathrm{s}} \sim 3 \mathrm{~m}_{\mathrm{b}}$ when $4.90<\mathrm{M}_{\mathrm{s}}<6.27$; and (3) $\mathrm{m}_{\mathrm{b}}=6.0$ when $6.27<\mathrm{M}_{\mathrm{s}}$. 
As mentioned above, the surface-wave magnitude is in the study range 4.2 to 7.8 . Obviously, Geller's results can only describe our observation when $4.20<\mathrm{M}_{\mathrm{s}}<4.90$ for small earthquakes, yet not for moderate and large events with $4.9 \leq \mathrm{M}_{\mathrm{s}} \leq 7.8$. Figure 5 in Geller (1976) is the basis for him to claim that his theoretical relations can fit the data. However, that figure shows high dispersion of data points, especially for those with $6.0 \leq \mathrm{M}_{\mathrm{s}} \leq 8.5$. From Haskell's model, Geller (1976) developed the scaling relations between source parameters on the basis of three similarity relations among five source parameters, i.e., the fault length (L), fault width (W), average dislocation (D), rupture velocity $(\beta)$, and rise time $(\tau)$. The three similarity relations are: (1) W/L = constant (aspect ratio); (2) $\mathrm{D} / \mathrm{L}=$ constant (strain drop); and (3) $\beta \tau / \mathrm{L}=$ constant (dynamic similarity). Kanamori and Anderson (1975) stressed that the first relation seems reasonable for most earthquakes; the second one is valid for large events; and the third does not hold for large events. However, the results by Wang and Ou (1998) implicated that the first similarity relation is valid for small events, yet not for large ones. These reasons might make the scaling relations developed by Geller (1976) questionable.

Figure 5 displays the plot of $\log \left(\mathrm{M}_{\mathrm{o}}\right)$ versus $\mathrm{M}_{\mathrm{L}}$. The data points are somewhat dispersive, even though they follow a linear trend. Regardless of the small number of data, the data points associated with $\mathrm{M}_{\mathrm{L}}>6.5$ depart from the solid line. This implies that the size of large earthquakes could be under-estimated when local magnitude is used. For the Taiwan earthquakes, the relation between $\mathrm{M}_{\mathrm{o}}$ and $\mathrm{M}_{\mathrm{L}}$ has been determined by others:

$$
\log \left(\mathrm{M}_{\mathrm{o}}\right)=(1.598 \pm 0.236) \mathrm{M}_{\mathrm{L}}+(14.571 \pm 1.683)
$$

by Wang et al. (1989); and

$$
\log \left(\mathrm{M}_{\mathrm{o}}\right)=(0.914 \pm 0.035) \mathrm{M}_{\mathrm{L}}+(19.043 \pm 0.533)
$$

by Li and Chiu (1989). Equations (14) and (15) are depicted with dashed and dotted lines, respectively, in Fig. 5. Obviously, these two lines depart from Equation (4) and cannot describe the data points well. These two lines lie somewhat below the solid line. This means that the local magnitude determined by Wang et al. (1989) and Li and Chiu (1989) is larger than that by Shin (1993). The difference in seismic moment between dotted and solid lines increases with $\mathrm{M}_{\mathrm{L}}$, while that between dashed and solid lines decreases with increasing $\mathrm{M}_{\mathrm{L}}$.

The $\mathrm{M}_{\mathrm{L}}$ scale is not restricted to a particular wave type or period. It is not easy to develop relations between $\mathrm{M}_{\mathrm{L}}$ and other source parameters. Nevertheless, Hanks and Boore (1984) constructed the correlation between $\mathrm{M}_{\mathrm{o}}$ and $\mathrm{M}_{\mathrm{L}}$ in three frequency ranges based on three particular frequencies, i.e., $\mathrm{f}_{\mathrm{s}}$ (the natural frequency of the Wood- Anderson seismograph, $\mathrm{f}_{\mathrm{o}}$ (the corner frequency), and $\mathrm{f}_{\max }$ [the cut-off frequency defined by Hanks (1982)]. The correlations are: (1) $\log \left(\mathrm{M}_{\mathrm{o}}\right) \sim 3.0 \mathrm{M}_{\mathrm{L}}$ as $\mathrm{f}_{\mathrm{o}}<<\mathrm{f}_{\mathrm{s}}$ (for large events); (2) $\log \left(\mathrm{M}_{\mathrm{o}}\right) \sim 1.5 \mathrm{M}_{\mathrm{L}}$ as $\mathrm{f}_{\mathrm{s}}<<\mathrm{f}_{\mathrm{o}}<\mathrm{f}_{\max }$ (for moderate events); (3) $\log \left(\mathrm{M}_{\mathrm{o}}\right) \sim 1.0 \mathrm{M}_{\mathrm{L}}$ as $\mathrm{f}_{\mathrm{o}}>\mathrm{f}_{\max }$ (for small events). Since most of events in use can be classified into large or moderate earthquakes, Eq. (4) cannot be interpreted by the theoretical relationships of Hanks and Boore (1984). They developed correlations on the basis of the instrumental response curve of the standard Wood-Anderson seismograph. While Shin 
(1993) used short-period digital seismograms recorded by S-13 and L-4C sensors to simulate the Wood-Anderson waveforms. The instrumental response curves of the two kinds of sensors are different from that of the standard Wood-Anderson seismograph, although the simulations are made based on the response curve of the Wood-Anderson seismograph, the resultant waveforms cannot be completely the same as the standard Wood-Anderson seismograms. Hence, the existence of a distinction between observed and theoretical relationships is inevitable.

It is interesting and necessary to compare $\mathrm{m}_{\mathrm{b}}$ and $\mathrm{M}_{\mathrm{L}}$. Both of them are determined from the peak amplitudes of seismograms at short periods: $\mathrm{m}_{\mathrm{b}}$ is estimated from the telemetered $\mathrm{P}$ waves around 1 second, while $\mathrm{M}_{\mathrm{L}}$ is based on the local or regional $\mathrm{S}$ waves or $\mathrm{Lg}$ waves. Figure 6 shows a linear trend of data points, even though they are somewhat dispersive. Three relations between $\mathrm{m}_{\mathrm{b}}$ and $\mathrm{M}_{\mathrm{L}}$ determined by others are:

$$
\mathrm{m}_{\mathrm{b}}=0.85 \mathrm{M}_{\mathrm{L}}+(0.27 \pm 0.60)
$$

by Shin (1986);

$$
\mathrm{M}_{\mathrm{L}}=(1.268 \pm 0.094) \mathrm{m}_{\mathrm{b}}-(0.604 \pm 0.485)
$$

by Wang et al. (1989); and

$$
\mathrm{M}_{\mathrm{L}}=0.75 \mathrm{~m}_{\mathrm{b}}+1.94 \text {, }
$$

by Cheng and Yeh (1989). Equations (16) - (18) are depicted with dashed, dotted and dotteddashed lines, respectively, in Fig. 6. Essentially, the three lines depart from Eq. (5) and lie below the data points. Obviously, the three relationships cannot interpret the data points well, implying that the ML used in the previous study is larger than that in this study. The dashed and dotted lines are somewhat in parallel and do not depart too much. The deviation between the two lines decreases with increasing $\mathrm{M}_{\mathrm{L}}$. The dotted-dashed line remarkable deviates from others.

The difference in $\mathrm{M}_{\mathrm{L}}$ may be caused by, at least, three reasons: the use of different attenuation functions, the different numbers of seismograms used in the determination of local magnitude and site amplification. Shin (1993) derived a new attenuation function for the calculation of local magnitude in the Taiwan area. He also obtained a relationship between new [denoted by $\mathrm{M}_{\mathrm{L}}(\mathrm{new})$ ] and old [denoted $\mathrm{M}_{\mathrm{L}}$ (old)] local magnitudes estimated, respectively, from new and old attenuation functions in the form:

$$
\mathrm{M}_{\mathrm{L}}(\text { new })=0.97 \mathrm{M}_{\mathrm{L}}(\text { old })+0.09
$$

If the $\mathrm{M}_{\mathrm{L}}$ used in this study is divided by 0.97 , however, most of the data points still lie above the lines of Equations (16) and (17). This indicates that the difference in $\mathrm{M}_{\mathrm{L}}$ is not simply caused by the use of different attenuation functions. The $\mathrm{M}_{\mathrm{L}}$ used in this study is the averaged value of local magnitudes measured from the simulated Wood-Anderson seismograms of all CWBSN stations (Shin 1993), while those in other studies are either estimated directly from 
seismograms recorded at a single simulated Wood- Anderson station (Shin 1986; Wang et al. 1989) or converted from the duration magnitude or other magnitudes (Cheng and Yeh 1989). This would result in deviations. In addition, the simulated Wood-Anderson seismograph of IES was installed in the Taipei basin where site amplification caused by unconsolidated sediments is high (Wen and Peng 1998; Chen 2003). Therefore, $\mathrm{M}_{\mathrm{L}}$ could be overestimated in the previous studies.

If we consider the calculation of $m_{b}$ to be consistently the same, Eq. (5) where $\mathrm{M}_{\mathrm{L}}$ is denoted by $\mathrm{M}_{\mathrm{L}}(C W B)$ and Eq. (16) where $\mathrm{M}_{\mathrm{L}}$ is denoted by $\mathrm{M}_{\mathrm{L}}(\mathrm{WA})$, then this leads to:

$$
\mathrm{M}_{\mathrm{L}}(\mathrm{CWB})=1.29 \mathrm{M}_{\mathrm{L}}(\mathrm{WA})-2.15 \quad\left[4.0 \leq \mathrm{M}_{\mathrm{L}}(\mathrm{WA}) \leq 6.6\right]
$$

Equation (20) can be used to unify the local magnitude for pre-1991 earthquakes determined from only a single Wood-Anderson seismogram $\left[\mathrm{M}_{\mathrm{L}}(\mathrm{WA})\right]$ in Taiwan and for post-1991 events routinely determined by the CWBSN $\left[\mathrm{M}_{\mathrm{L}}(\mathrm{CWB})\right]$.

Figure 7 shows the plot of $M_{s}$ versus $M_{L}$. The data points follow a linear trend, but they are somewhat dispersive. Like Figure 5, some data points associated with $\mathrm{M}_{\mathrm{L}}>6.5$ depart from the solid line. This implies that the size of large earthquakes could be under-estimated when the local magnitude is used.

\section{CONCLUSIONS}

According to the seismic moments and magnitudes for earthquakes occurring in the Taiwan region published in the Global CMT catalog, the $\mathrm{M}_{\mathrm{o}}-\mathrm{M}_{\mathrm{s}}, \mathrm{M}_{\mathrm{o}}-\mathrm{m}_{\mathrm{b}}$, and $\mathrm{M}_{\mathrm{s}}-\mathrm{m}_{\mathrm{b}}$ relationships are estimated. The inferred relationships have good agreement with Wang's results (1985). Meanwhile, the inferred relationships do not depart from Nuttli's results (1985) too much. This might imply that the tectonic conditions, accumulation of earthquake energy, and source properties of the Taiwan region behave like the average of the circum-Pacific seismic belt. For large earthquakes used in this study, the value of local magnitude could be underestimated. The local magnitude, used in previous studies, was determined from only a single simulated Wood-Anderson seismograph of IES at the Taipei Basin. Its value was overestimated due to site amplification caused by unconsolidated sediments.

Acknowledgements Helpful comments from Dr. G. B. Ou and an anonymous reviewer greatly improved the manuscript. This work was sponsored by Academia Sinica under Grant No. AS94-TP-A08.

\section{REFERENCES}

Aki, K., 1966: Generation and propagation of G waves from the Niigata earthquake of June 16, 1964. Bull. Earthquake Res. Inst. (Tokyo Univ.), 44, 23-88.

Aki, K., 1967: Sealing law of seismic spectrum. J. Geophys. Res., 72, 1217-1232. 
Aki, K., 1972: Scaling law of earthquake source time-function. Geophys. J. R. Astro. Soc., 31, 3-25.

Ben-Menahem, A., S. J. Singhand, and F. Solomon, 1969: Static deformation of a spherical earth model by internal dislocation. Bull. Seismol. Soc. Am., 59, 813-853.

Chen, K. C., and J. H. Wang, 1988: A study on aftershocks and focal mechanisms of two 1986 earthquakes in Hualien, Taiwan. Proc. Geol. Soc. China, 31, 65-72.

Chen, K. C., 2003: Strong ground motion and damage in the Taipei basin from the Moho reflected seismic waves during the March 31, 2002, Hualien, Taiwan earthquake. Geophys. Res. Lett., 30, doi:10.1029/2003GL017193.

Cheng, S. N., and Y. T. Yeh, 1989: Catalog of earthquakes in Taiwan from 1604 to 1988. Open File Rept., Inst. Earth Sci., Acad. Sin., 255 pp. (in Chinese)

Chung, D. H., and D. L. Bernreuter, 1981: Regional relationships among earthquake magnitude scales. Rev. Geophys. Space Phys., 19, 649-663.

Ekstrom, G., and A. M. Dziewonski, 1988: Evidence of bias in estimations of earthquake size. Nature, 322, 319-323.

Geller, R. J., 1976: Scaling relations for earthquake source parameters and magnitudes. Bull. Seismol. Soc. Am., 66, 1501-1523.

Gutenberg, B., 1945: Amplitudes of surface waves and magnitudes of shallow earthquakes. Bull. Seismol. Soc. Am., 35, 3-12.

Gutenberg, B., and C. F. Richter, 1956: Magnitude and energy of earthquakes. Ann. Geofis., 9, $1-15$.

Hanks, T. C., and H. Kanamori, 1979: A moment magnitude scale. J. Geophys. Res., 84, 2348-2350.

Hanks, T.C., 1982: $\mathrm{f}_{\max }$. Bull. Seismol. Soc. Am., 72, 1867-1879.

Hanks, T. C., and D. M. Boore, 1984: Moment-magnitude relations in theory and practice. $J$. Geophys. Res., 89, 6229-6235.

Haskell, N., 1964: Total energy and energy spectral density of elastic wave radiation from propagating faults. Bull. Seismol. Soc. Am., 56, 1811-1842.

Huang, M. W., and J. H. Wang, 2002: Scaling of displacement spectra of near-fault seismograms of the 1999 Chi-Chi, Taiwan, earthquake. Geophys. Res. Lett., 29, 47:1-4.

Hwang, R. D., J. H. Wang, B. S. Huang, K. C. Chen, W. G. Huang, T. M. Chang, R. D. Huang, H. C. Chiu, and C. C. P. Tsai, 2001: Estimates of stress drop from near-field seismograms of the $\mathrm{M}_{\mathrm{s}}$ 7.6 Chi-Chi, Taiwan, earthquake of September 20, 1999. Bull. Seismol. Soc. Am., 91, 1158-1166.

Kanamori, H., and D. L. Anderson, 1975: Theoretical basis of some empirical relations in seismology. Bull. Seismol. Soc. Am., 65, 1073-1095.

Koyama, J., 1994: General description of the complex faulting process and some empirical relations in seismology. J. Phys. Earth, 42, 103-148.

Li, C., and H. C. Chiu, 1989: A simple method to estimate the seismic moment from seismograms. Proc. Geol. Soc. China, 32, 197-207.

Lin, C. H., 2002: Active continental subduction and crustal exhumation: The Taiwan orogeny. Terra Nova, 14, 281-287. 
Liu, C. C., 1981: The simulation of Wood-Anderson seismograph by using a L-4C geophone and integrated circuit. Inst. Earth Sci., Acad. Sin., unpublished. (in Chinese)

Ma, K. F., C. T. Lee, Y. B. Tsai, T. C. Shin, and J. Mori, 1999: The Chi-Chi, Taiwan earthquake: Large surface displacements on an inland thrust fault. EOS, Trans., AGU, 80, 605-611.

Miyamura, S., 1978: Magnitude of earthquakes (I). IISEE Lecture Notes No. 11, Int. Inst. Seismol. Earthquake Engin., Japan, 83 pp.

Miyamura, S., 1982: On the importance of continuity of magnitude scales. Tectonophysics, 84, 47-55.

Nortman, R., and S. J. Duda, 1983: Determination of spectral properties of earthquakes from their magnitudes. Tectonophysics, 93, 251-275.

Nuttli, O. W., 1983a: Empirical magnitude and spectral scaling relations for mid-plate and plate-margin earthquakes. Tectonophysics, 93, 207-223.

Nuttli, O. W., 1983b: Average seismic source-parameter relations for mid-plate earthquakes. Bull. Seismol. Soc. Am., 73, 519-535.

Nuttli, O. W., 1985: Average seismic source-parameter relations for plate-margin earthquakes, preprint. Tectonophysics, 118, 161-174.

Purcaru, G., and H. Berckhemer, 1978: A magnitude scale for very large earthquakes. Tectonophysics, 49, 189-198.

Richter, C. F., 1935: An instrumental earthquake magnitude scale. Bull. Seismol. Soc. Am., 25, 1-32.

Shin, T. C., 1986: Duration-magnitude correction for Taiwan Telemetered Seismographic Network. Bull. Inst. Earth Sci., Acad. Sin., 6, 109-120.

Shin, T. C., 1993: The calculation of local magnitude from the simulated Wood-Anderson seismograms of the short-period seismograms in the Taiwan area. Terr. Atmos. Ocean. Sci., 4, 155-170.

Tsai, Y. B., T. L. Teng, J. M. Chin, and H. L. Lin, 1977: Tectonic implications of the seismicity in the Taiwan region. Memoir Geol. Soc. China, 2, 13-41.

Wang, J. H., 1985: Seismic moments and magnitudes of 16 moderate Taiwan earthquakes. Proc. Geol. Soc. China, 28, 177-185.

Wang, J. H., C. C. Liu, and Y. B. Tsai, 1989: Local magnitude determined from a simulated Wood-Anderson seismograph. Tectonophysics, 166, 15-26.

Wang, J. H., 1992: Magnitude scales and their relations for Taiwan earthquakes: A review. Terr. Atmos. Ocean. Sci., 3, 449-468.

Wang, J. H., 1998: Studies of earthquake seismology in Taiwan during the 1897-1996 period. J. Geol. Soc. China, 41, 291-336.

Wang, J. H., and S. S. Ou, 1998: On scaling of earthquake faults. Bull. Seismol. Soc. Am., 88, 758-765.

Wang, J. H., M. W. Huang, K. C. Chen, R. D. Hwang, and W. Y. Chang, 2002: Aspects of characteristics of near-fault ground motions of the 1999 Chi-Chi (Taiwan) earthquake. J. Chin. Inst. Engin., Series A, 25, 507-519.

Wang, J. H., 2006a: A review of the source parameters of the 1999 Chi-Chi, Taiwan, earthquake. Terr. Atmos. Ocean. Sci., 17, 179-202. 
Wang, J. H., 2006b: Energy release and heat generation during the 1999 Chi-Chi, Taiwan, earthquake. J. Geophys. Res., 111, B11312, doi:10.1029/2005JB004018.

Wen, K. L., and H. Y. Peng, 1998: Site effect analysis in the Taipei basin: Results from TSMIP network data. Terr. Atmos. Ocean. Sci., 9, 691-704.

Wu, F. T., 1978: Recent tectonics of Taiwan. J. Phys. Earth, 26, Suppl., S265-S299.

Yeh, Y. T., G. B. Ou, and C. C. Lin, 1982: Determination of local magnitude scale for Taiwan. Bull. Inst. Earth Sci., Acad. Sin., 2, 37-48.

Yeh, Y. T., and P. S. Hsu, 1985: Catalogue of strong earthquakes in the Taiwan region from 1644 to 1984. Inst. Earth Sci., Acad. Sin., unpublished. (in Chinese)

Chen, K. C., W. G. Huang, and J. H. Wang, 2007: Relationships among magnitudes and seismic moment of earthquakes in the Taiwan region. Terr. Atmos. Ocean. Sci., 18, 951-973, doi: 10.3319/TAO.2007.18.5.951(T). 